\title{
Quantum Computers and Quantum Algorithms. Part 2. Quantum Algorithms
}

\section{M. Solovyev}

Solovyev Vladimir Mihajlovich, Saratov State University, 83, Astrakhanskaya st., Saratov, Russia, 410012, svm@sgu.ru

The paper discusses principles of construction for quantum algorithms and their main features. Distinction of quantum parallelism from classical methods of high-performance computing is shown. Quantum algorithms design strategy is presented based on quantum circuits. Methods of programming for implementation of quantum algorithms using high-level languages are proposed. An approach to implement unitary transformations based on the oracle method is described.

Key words: quantum computing, quantum computers, quantum algorithms, qubit, quantum gate, quantum superposition, quantum entanglement, quantum parallelism, quantum interference, oracle, quantum-programming languages.

\section{References}

1. Solovyev V. M. Quantum Computers and Quantum Algorithms. Pt. 1 : Quantum Computers. Izv. Saratov Univ. (N.S.), Ser. Math. Mech. Inform., 2015, vol. 15 , iss. 4, pp. 462-477. DOI: 10.18500/18169791-2015-15-4-462-477 (in Russian).

2. Algebraic and Number Theoretic Algorithms. Available at: http://math.nist.gov/ quantum/zoo/ (accessed 23 June 2015).

3. Bogdanov U. I., Kokin A. A., Lukichev V. F., Orlikovskij A. A., Semenihin I. A., Chernavskij A. U. Quantum mechanics and the development of information technology. Information technologies and computer systems, 2012, no. 1, pp. 17-31 (in Russian).

4. Venegas-Andraca S. E. Quantum Walks for Computer Scientists. Synthesis Lectures on Quantum Computing, Morgan Claypool, 2008, 133 p.

5. Gorbachev V. N., Zhiliba A. I. Physical basis of modern information processes or textbook on quantum teleportation, quantum computing and other issues of quantum information. Tver, Tver State University, 2001, 43 p. (in Russian).
6. Shor P. W. Polynomial-time Algorithms for Prime Factorization and Discrete Logarithms on a Quantum Computer. arXiv: quant-ph/9508027, 25.01.1996, $28 \mathrm{p}$.

7. Williams C. P. Explorations in Quantum Computing. Springer-Verlag London Ltd., 2011, 740 p.

8. Closing in on quantum computing. Available at: http://www.wired.com/2014/10/quantumcomputing-close (accessed 23 June 2015).

9. Bhambri S. Quantum Clouds: A future perspective. arXiv: quant-ph/1410.6502v1, 05.10.2014, 14 p.

10. Valiev K. A. Quantum computers and quantum computing. Uspekhi Fizicheskikh Nauk [Successes of physical sciences], 2005, vol. 175, no 1, pp. 3-39 (in Russian).

11. Metodi T. S., Faruque A. I., Chong F. T. Quantum Computing for Computer Architects. Synthesis Lectures on Computer Architecture, Morgan Claypool, 2011, 203 p.

\section{ОБ ОДНОМ ПОДХОДЕ К НЕЧЕТКОМУ ЛОГИЧЕСКОМУ МОДЕЛИРОВАНИЮ ЦИФРОВЫХ УСТРОЙСТВ}

\begin{abstract}
Д. В. Сперанский
Сперанский Дмитрий Васильевич, доктор технических наук, просрессор кафредры высшей и прикладной математики, Московский государственный университет путей сообщения, Speranskiy.dv@ gmail.com

В статье исследуется проблема двоичного нечеткого моделирования цифрровых устройств (ЦУ). В отличие от аналогичной классической проблемы предполагается, что входные сигналы ЦУ являются нечеткими. В реальных ЦУ для каждого входа (0 или 1) существует определенный диапазон в вольтах. Если входной сигнал выходит за этот диапазон, то корректность его идентисрикации не гарантируется. Нечеткость входного сигнала означает, что наблюдаемые его значения могут быть либо внутри определенного диапазона, или вне его. Известно, что логическое моделирование каждого цУ состоит в вычислении значения определенного логического выражения. Это выражение есть математическая модель ЦУ. Кроме того, это логическое выражение может всегда быть представлено в терминах трех логических операций, а именно И, ИЛИ, НЕ. В статье предлагается метод сведения исследуемой проблемы к проблеме нечеткого моделирования систем в пространстве вещественных чисел. Метод основан на представлении логического выражения с исполь-
\end{abstract}


зованием бесконечнозначной (непрерывной) логики. Вычисление в этой логике сводится к вычислению выражения в пространстве вещественных чисел. Предложенная в статье процедура намного менее трудоемка, чем ранее известная процедура для нечеткого моделирования, использующая нечеткую арифрметику в пространстве вещественных чисел.

Ключевые слова: непрерывная логика, цифрровые устройства, нечеткое логическое моделирование.

DOI: 10.18500/1816-9791-2016-16-1-112-119

\section{ВВЕДЕНИЕ}

Как известно, логическое моделирование цифровых устройств (ЦУ) широко применяется для решения многих задач, к числу которых относится, в частности, проектирование ЦУ, включающее разработку структурной и функциональной схем ЦУ, анализ схемотехнической реализации, разработку его принципиальной электрической схемы и т.д. Упомянутое моделирование может осуществляться с различной степенью детализации, которая существенно зависит от преследуемой при моделировании цели.

Напомним, что обычно логическое моделирование ЦУ основано на применении классического математического аппарата, подразумевающего выполнение операций с точными значениями данных. Вместе с тем для реальных ЦУ такие данные часто являются неточными (нечеткими) по объективным причинам (неточность измерений, нечеткость в описании функционирования ЦУ и т. п.). Очевидно, что это приводит к огрублению результатов моделирования. Понятно, что для отражения нечеткости данных нужно иметь подходящие средства, созданию которых способствовало введение Л. Заде [1] концепции нечетких множеств.

Названная концепция послужила толчком к появлению и развитию таких новых разделов математики и ее приложений, как нечеткая арифметика, нечеткая алгебра, нечеткая логика, теория нечеткого управления и т. п. Не останавливаясь на обзоре работ в этих направлениях, поскольку это лежит за рамками нашей статьи, назовем лишь некоторые из известных публикаций [2-5], связанных с рассматриваемыми ниже вопросами.

В предлагаемой статье рассматривается нечеткое двоичное логическое моделирование ЦУ, ориентированное, в частности, на решение задач контроля и диагностирования ЦУ, идентификации ЦУ и т. п.

\section{1. ПОСТАНОВКА ЗАДАЧИ}

В качестве ЦУ как комбинационных, так и с памятью далее рассматриваются устройства, выходные сигналы которых по заданным входным сигналам вычисляются с использованием логических выражений. Это означает, что совокупность таких выражений представляет собой математическую модель рассматриваемого ЦУ. Предполагается, что каждое логическое выражение в общем случае может содержать различные логические операции, включая отрицание, конъюнкцию, дизъюнкцию, сумму по модулю 2 и др. Известно [6], что, например, первые три названные операции образуют полную систему булевых функций (базис), т. е. любая другая логическая функция может быть представлена в виде логического выражения, содержащего только функции из этого базиса.

Поскольку реальные ЦУ комбинационного типа чаще всего состоят из микросхем, реализующих функции упомянутого базиса, математической моделью таких ЦУ являются описывающие их функционирование логические выражения. Известно также [7], что любое ЦУ с памятью всегда можно преобразовать в эквивалентную ему итеративную комбинационную сеть. Таким образом, и для ЦУ с памятью соответствующее ему логическое выражение также является адекватной математической моделью. Исходя из сказанного, далее для простоты изложения в качестве объекта исследования будет рассматриваться комбинационное ЦУ (хотя это и не принципиально) с несколькими входами и одним выходом.

В классической модели ЦУ при традиционном двоичном логическом моделирования на каждом его входном и выходном полюсе может появиться только точный двоичный сигнал «0» или «1». Исследуемая здесь задача предполагает, что поступающие на входы ЦУ сигналы являются нечеткими. Понятно, что получаемые при этом выходные сигналы ЦУ также являются нечеткими. 
Сформулируем теперь рассматриваемую нами задачу. Пусть заданы логическое выражение, реализуемое исследуемым ЦУ, и входной набор ЦУ, состоящий из нечетких сигналов. На основе этой информации требуется осуществить нечеткое логическое моделирование ЦУ и определить двоичное значение выходного сигнала (0 или 1), являющееся максимальным приближением к вычисленному в результате моделирования нечеткому значению.

Использованные выше термины «нечеткий сигнал» и «нечеткое моделирование» требуют уточнения. Напомним [8], что использование логической модели ЦУ предполагает некоторую идеализацию реальных сигналов. Поведение каждого из реальных элементов ЦУ описывается в терминах переменных, отображающих значения меняющихся во времени физических величин. При этом у значения переменной всегда имеется область неопределенности, обусловленная инерционностью элементов. На диаграммах изменения сигнала это изображается трапецеидальной его формой, а не абсолютно прямоугольной.

Так, изменение сигнала от одного уровня до другого (за время переходного процесса) в действительности различается и составляет, как правило, приблизительно 10\% при изменении $0 \rightarrow 1$ и $90 \%$ при изменении $1 \rightarrow 0$ от максимального значения сигнала. Уровни сигнала (0 и 1) должны находиться в некоторых заранее допустимых пределах и при выходе за эти пределы сигнал иногда не может быть отождествлен ни с 1, ни с 0. Заметим, что в ЦУ значения сигналов отображаются соответствующими уровнями напряжения. Так, например, в КМОП-схемах это $0.0 \div 1.5$ В для отображения сигнала 0 и $3.5 \div 5.0$ В для сигнала 1 ; в ТТЛ-схемах для тех же сигналов эти уровни таковы: $0.0 \div 0.8$ В и $2.0 \div 5.0 \mathrm{~B}$.

\section{2. ПРИЛОЖЕНИЕ НЕПРЕРЫВНОЙ ЛОГИКИ К НЕЧЕТКОМУ ЛОГИЧЕСКОМУ МОДЕЛИРОВАНИЮ ЦУ}

Классическая двузначная логика во многих случаях является недостаточной, что привело к разработке рядом авторов (Лукасевичем, Постом, Тарским, Яблонским и др.) так называемых многозначных логик. Такие логики относятся к разряду дискретных логик, в которых имеется конечное множество значений истинности высказывания на заданном отрезке числовой оси. При осуществлении предельного перехода, заключающегося в устремлении к бесконечности числа значений истинности высказывания, в итоге получаем бесконечнозначную логику, называемую также непрерывной логикой. Таким образом, непрерывную логику можно рассматривать как обобщение дискретной логики.

Основная идея предлагаемого ниже подхода к нечеткому логическому моделированию базируется на применении для этой цели математического аппарата нечеткой логики.

Отметим, что приложениям непрерывной логики к исследованию динамики, надежности и других проблем ЦУ посвящен целый ряд работ В. И. Левина, среди которых назовем, например, $[9,10]$. Решение некоторых из перечисленных проблем требует применения моделирования. Однако такое моделирование предполагает исследование функционирования ЦУ, во-первых, в динамике, когда на его входы подаются последовательности символов, а не отдельные символы, и, во-вторых, когда входные воздействия являются точными (четкими). Рассматриваемая нами задача отличается наличием нечетких входных воздействий и необходимостью вычисления реакции ЦУ только на отдельные входные воздействия. Понятно, что такая «статическая» задача менее сложна и есть основания рассчитывать на разработку более простого метода ее решения, чем применение достаточно сложного и трудоемкого аппарата «динамического» моделирования.

Напомним некоторые положения непрерывной логики, с основами которой можно ознакомиться, например, по книге С. А. Гинзбурга [11]. Значения истинности в этой логике образуют ограниченное множество вещественных чисел, содержащее свою верхнюю грань $B$ и нижнюю грань $A$. Предполагается, что это множество является симметричным относительно точки $C=(A+B) / 2$, которую можно назвать центром множества значений высказываний. Основными логическими операциями в непрерывной логике являются обобщенные дизъюнкция, конъюнкция и отрицание - аналоги соответствующих операций в классической двузначной логике. Значения истинности перечисленных обобщенных операций определяются следующим образом:

$$
\begin{aligned}
& x \vee y=\max (x, y), \\
& x \wedge y=\min (x, y),
\end{aligned}
$$




$$
\bar{x}=A+B-x=2 C-x,
$$

где $x$ и $y-$ значения истинности соответствующих высказываний. Формула (3) означает, что значения истинности двух противоположных высказываний представляют собой две точки на отрезке $[A, B]$, расположенные симметрично относительно центра множества значений.

Отметим, что для непрерывной логики так же, как и для двузначной логики, оказываются справедливыми следующие общие законы:

$$
\begin{array}{rlr}
x \vee x=x, & x \wedge x=x & \text { (тавтология); } \\
x \vee y=y \vee x, & x \wedge y=y \wedge x & \text { (коммутативность); } \\
(x \vee y) \vee z=x \vee(y \vee z), & (x \wedge y) \wedge z=x \wedge(y \wedge z) & \text { (ассоциативность); } \\
x \wedge(y \vee z)=(x \wedge y) \vee(x \wedge z), & x \vee(y \wedge z)=(x \vee y) \vee(x \vee z) & \text { (дистрибутивность); } \\
\overline{x \vee y}=\bar{x} \wedge \bar{y}, & \overline{x \wedge y}=\bar{x} \vee \bar{y} & \text { (законы де Моргана). }
\end{array}
$$

Помимо приведенных законов для непрерывной логики справедливыми являются также следующие:

$$
\begin{array}{ll}
x \vee A=x, & x \wedge B=x, \\
x \vee B=x, & x \wedge A=A .
\end{array}
$$

Понятно, что поскольку аргументами логических функций непрерывной логики являются непрерывные величины, то и значениями таких функций также являются непрерывные величины. Поэтому естественно, что логические операции могут здесь сочетаться с операциями обычной алгебры - сложением, умножением, вычитанием. Такое в принципе возможно и для дискретной логики, но для нее результаты выполнения логических и алгебраических операций должны обязательно принадлежать множеству принятых дискретных значений. Что касается непрерывной логики, то в ней результат операций должен удовлетворять единственному ограничению - находиться в пределах отрезка $[A, B]$.

Очевидно, что при сочетании логических операций с алгебраическим суммированием и умножением оказываются справедливыми некоторые законы, на которых мы здесь не останавливаемся, поскольку их приложения лежат за рамками нашей статьи.

\section{3. СТРУКТУРНАЯ МОДЕЛЬ НЕЧЕТКОГО ЛОГИЧЕСКОГО МОДЕЛИРОВАНИЯ}

Проблемы нечеткого моделирования, необходимость исследования которых возникла с 70-х годов прошлого века в связи с появлением обширных приложений теории нечетких множеств, сейчас оказались в зоне интересов многих ученых. Значительное внимание было проявлено в первую очередь к нечетким моделям процессов, описываемых различного рода уравнениями и соотношениями, включающими переменные со значениями из поля вещественных чисел. Соответствующие методы и многие аспекты такого моделирования в настоящее время достаточно хорошо разработаны. В частности, проблемы и методы нечеткого моделирования в поле вещественных чисел подробно изложены в монографии А. Пегата [12], терминология и понятия из которой используются нами в дальнейшем изложении.

Как известно [12], нечеткое моделирование в поле вещественных чисел потребовало разработки нечеткой арифметики (определения всех четырех арифметических действий над нечеткими числами), нечеткой математики (определения операторов объединения, пересечения, понятий нечеткого отношения и импликации). Кроме того, значительных усилий потребовала разработка структуры и операций в нечетких моделях и различных их типов, и, наконец, методов нечеткого моделирования. Результатом указанных разработок явилось создание достаточно эффективной, но весьма трудоемкой в применении математической теории.

Для решения рассматриваемой нами задачи упомянутая выше теория безусловно может быть использована. Вместе с тем специфика этой задачи позволяет уменьшить в значительной степени трудоемкость процесса нечеткого логического моделирования ЦУ, теоретически обоснованного в [12]. Так, этому способствует отсутствие необходимости использования нечеткой арифметики, аппарата 
нечеткой математики и возможность значительного упрощения типовой структуры нечеткой модели, описанной в [12]. Остановимся подробнее на отмеченных особенностях.

Условимся, что используемая нами математическая модель ЦУ (логическое выражение, описывающее его функционирование) содержит переменные, представленные в единицах электрического напряжения (в вольтах). Конкретные значения переменные приобретают в результате измерений в процессе штатного функционирования ЦУ и являются нечеткими.

Проиллюстрируем предлагаемый метод на примере ТТЛ-схемы. Как было отмечено выше, сигналу «0» в таких схемах соответствует диапазон напряжения $0 \div 0.8$ В, а сигналу «1» - диапазон $2.0 \div 5.0 \mathrm{~B}$. Между этими диапазонами расположена так называемая мертвая зона, в пределах которой не гарантируется корректное восприятие уровня сигнала. Для ТТЛ-схем это диапазон $0.8 \div 2.0$ В. Середина этой зоны (в нашем случае напряжение 1.4 В) является так называемым пороговым уровнем сигнала. Если сигнал ниже этого уровня, то он может восприниматься как «0», если выше, то как «1» или как некорректный.

Условимся, что при нечетком логическом моделировании ЦУ на его входы подаются сигналы из двух нечетких множеств $A_{0}=$ «примерно $0 »$ и $A_{1}=$ «примерно 1». Пусть графики функций принадлежности этих множеств есть ломаные, изображенные на рисунке.

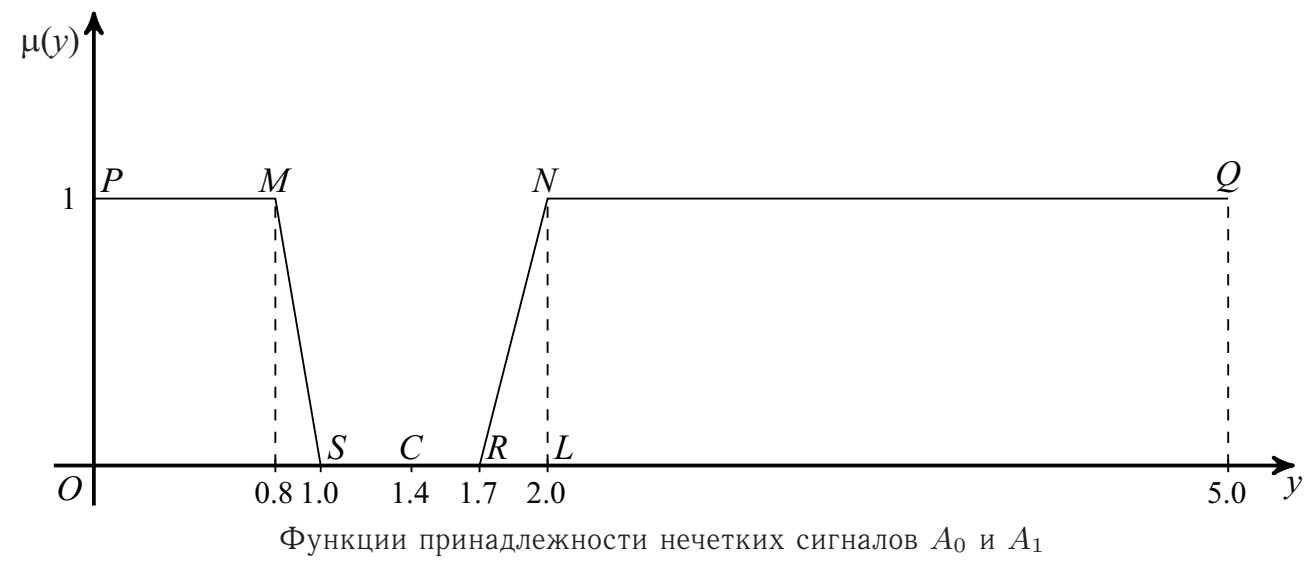

Ломаная PMS на рисунке соответствует множеству $A_{0}$, а ломаная QNR - множеству $A_{1}$. По оси абсцисс выписаны напряжения, определяющие эти множества и мертвую зону [0.8 $\div 2.0]$.

Перейдем к интерпретации перечисленных величин применительно к нашей задаче в терминах непрерывной логики. Значения возможных значений на выходе ЦУ образуют ограниченное множество вещественных чисел, лежащих на отрезке $[0.0 ; 5.0]$. Зону «нечеткости» входных и выходных сигналов ЦУ образуют значения, лежащие на отрезке $[A, B]$, где $A=0.8$ и $B=2.0$. Центром этого множества является точка $C=(A+B) / 2=(0.8+2.0) / 2=1.4$, которая выполняет роль порогового значения сигнала. Степень принадлежности $\mu(y)$ сигнала $y$ множествам $A_{0}$ и $A_{1}$, где $0 \leqslant \mu(y) \leqslant 1$, определяется с помощью функции принадлежности, графически представленной на рисунке.

Для выполнения нечеткого логического моделирования воспользуемся типовой структурой нечеткой модели, ориентированной на применение в поле вещественных чисел, которая детально описана в [12, гл. 5]. Напомним, что структура этой модели в общем случае состоит из трех последовательно соединенных блоков, выполняющих следующие функции.

1. Блок фазификации (fuzzification) для поступающих на него нечетких значений входов вычисляет их степени принадлежности $\mu(y)$ входным нечетким множествам.

2. Блок вывода (inference) на входе получает степени принадлежности, вычисленные предыдущим блоком, и на выходе вычисляет так называемую результирующую функцию $\mu_{r e s}(y)$ принадлежности выходного значения сигнала модели. Для выполнения вычислений этот блок включает в себя базу правил, механизм вывода и функции принадлежности выходного значения у.

3. Блок дефазификации (defuzzification) на основе $\mu_{r e s}(y)$ вычисляет значение $y^{*}$ выхода по его нечетким входным значениям. Этот блок включает в себя вычисление степеней выполнения условий отдельных правил, определения активизированных функций принадлежности заключений этих правил и результирующей функции принадлежности вывода из всех правил, входящих в базу. 
Имеющийся опыт проведения нечеткого моделирования ряда реальных процессов, описанных в литературе, подтверждает высокую трудоемкость реализации такого моделирования. Основную часть в нем составляют затраты на реализацию алгоритмов в блоках вывода и дефазификации.

Перейдем теперь к описанию предлагаемой нами процедуры нечеткого логического моделирования ЦУ, существенно менее трудоемкой по сравнению с трудоемкостью реализации приведенной выше типовой структурной модели.

Суть этой процедуры кратко изложим на примере простого комбинационного устройства с тремя входами $x_{1}, x_{2}, x_{3}$, значение на выходе которого описывается следующей структурной формулой:

$$
y=\left(x_{1} \wedge \bar{x}_{2}\right) \vee\left(x_{2} \wedge x_{3}\right) .
$$

Используя равенства (1)-(3), представим логическое выражение (4) в терминах обобщенных операций инверсии, конъюнкции и дизъюнкции:

$$
y=\max \left(\min \left(x_{1}, A+B-x_{2}\right), \min \left(x_{2}, x_{3}\right)\right) .
$$

При вычислении значения сигнала $у$ на выходе ЦУ при конкретных значениях нечетких переменных $x_{1}, x_{2}, x_{3}$, являющихся вещественными числами, применяются стандартные правила арифметики вещественных чисел для нахождения максимума и минимума. Результат этих вычислений есть нечеткое вещественное число. Описанные действия применительно к предлагаемой нами процедуре моделирования эквивалентны действию блоков фазификации и вывода типовой структуры нечеткой модели.

Следующий этап предлагаемой процедуры эквивалентен блоку дефазификации типовой структуры нечеткой модели. Сформулируем теперь предлагаемые нами правила, по которым нечеткое значение выхода ЦУ, полученное на предыдущем этапе при вычислении по формуле типа (5), преобразуется в четкое значение (0 или 1).

Предварительно введем следующие обозначения: $\Delta(0)=[0.0 ; 1.0]$ - диапазон значений сигнала, воспринимаемого как «примерно $0 » ; \Delta(1)=[1.7 ; 5.0]-$ диапазон значений сигнала, воспринимаемого как «примерно 1».

Прокомментируем эти диапазоны. Если нечеткое значение выхода ЦУ $y \in[0.0 ; 0.8]$, то оно воспринимается как четкий 0 , если же $y \in[0.8+\varepsilon ; 1.0]$, где $0<\varepsilon<0.2$, то по графику функции принадлежности определяется его степень $\mu(y)$ принадлежности сигналу «примерно 0». Аналогичная ситуация имеет место и для диапазона $\Delta(1)$. Если нечеткое значение выхода ЦУ $y \in[2.0 ; 5.0]$, то оно воспринимается как четкая 1 , если же $y \in[2.0-\delta ; 5.0]$, где $0<\delta<0.3$, то по графику функции принадлежности определяется его степень $\mu(y)$ принадлежности сигналу «примерно 1».

Будем предполагать, что экспертами-технологами в сфере производства ЦУ названы и обоснованы два значения $\mu^{*}(y)$ и $\bar{\mu}(y)$ из диапазона $[0 ; 1]$, являющиеся пороговыми значениями степени принадлежности сигналам соответственно «примерно 0» и «примерно 1». Это означает, если сигнал $y \in \Delta(0)$ $(y \in \Delta(1))$ со степенью принадлежности $\mu(y)$, где $\mu(y)<\mu^{*}(y)(\mu(y)<\bar{\mu}(y))$, то этот сигнал будет восприниматься как некорректный, в противном случае - как 0 (как 1).

Отметим, что экспертные оценки, аналогичные упомянутым выше, необходимы и для назначения правой (левой) границы отрезка $\Delta(0)(\Delta(1))$.

В терминах этих обозначений сформулируем правила, по которым нечеткое значение на выходе ЦУ, полученное по формуле типа (5), будет преобразовано в четкое значение (0 или 1).

1. Если нечеткое значение $y$ выхода ЦУ превышает пределы диапазона $\Delta(0)(\Delta(1))$ и $y<C$ $(y>C)$, где - пороговое значение сигнала, то этот сигнал считается некорректным, т.е. не воспринимаемым ни как 0 , ни как 1 .

2. Пусть нечеткое значение выхода ЦУ $y \in[0.0 ; 1.0]$ и пусть степень его принадлежности сигналу «примерно 0», определяемая по графику (см. рисунок), равно $\mu(y)$. Если $\mu(y) \geqslant \mu^{*}(y)$, то четкое значение сигнала $y$ полагается равным 0, в противном случае этот сигнал считается некорректным.

3. Пусть нечеткое значение выхода ЦУ $y \in[1.7 ; 5.0]$ и пусть степень его принадлежности сигналу «примерно 1», определяемое по графику (см. рисунок), равна $\mu(y)$. Если $\mu(y) \geqslant \bar{\mu}(y)$, то четкое значение сигнала $y$ полагается равным 1, в противном случае этот сигнал считается некорректным.

Фигурирующие в приведенных правилах величины $\mu^{*}(y)$ и $\bar{\mu}(y)$ - это своего рода пороговые степени принадлежности нечеткого выходного сигнала ЦУ, которые служат для отделения корректных 
сигналов от некорректных. Отметим, что конкретные значения этих величин диктуются спецификой различных технологий изготовления микросхем. Аналогичные соображения имеют место при назначении правой (левой) границы диапазона $\Delta(0)(\Delta(1))$.

Сравнение описанной нами процедуры нечеткого логического моделирования ЦУ с типовой структурой нечеткой модели, представленной в [12], позволяет сделать вывод о существенно меньшей трудоемкости процедуры, предложенной нами. Действительно, в нашей процедуре отсутствуют блоки фазификации и вывода, тогда как последний блок в типовой модели представляет собой наиболее трудоемкую ее часть. Кроме того, преобразование нечеткого значения выходного сигнала в четкое выполняется по предельно простым правилам в отличие от трудоемких преобразований, необходимых в типовой структурной модели.

Известно, что в общем случае адекватность разрабатываемых алгоритмов нечеткого моделирования в поле вещественных чисел и трудоемкость их реализации на основе структурной типовой модели зависит от многих факторов [12]. К их числу относятся формы представления нечетких чисел, определения арифметических операций над ними, выбор способов дефазификации и т.д. В нашей процедуре все эти факторы либо вообще отсутствуют, либо предельно упрощаются.

Качество алгоритмов нечеткого моделирования вполне естественно оценивать по соотношению числа правильных и неправильных результатов, получаемого путем сравнения реальных выходов ЦУ с вычисленными по оцениваемым алгоритмам в процессе проводимых численных или натурных экспериментов. Понятно, что от любого такого алгоритма невозможно требовать, чтобы на любых входных данных он всегда давал правильный результат, поскольку он оперирует с нечеткими данными. Для описанного в статье алгоритма нечеткого логического моделирования ЦУ, как нетрудно убедиться с помощью простых рассуждений и вычислений, для всех входных наборов, когда сигналы 0 и 1 представлены их заявленными по технологии диапазонами напряжений, получаются правильные результаты. Вместе с тем на аномальных входных воздействиях, когда они находятся вне пределов их заданных по технологии диапазонов, гарантии получения только правильных результатов отсутствуют. Понятно, что соотношение числа верных и неверных результатов, получаемых при нечетком логическом моделировании по предложенной процедуре, в общем случае зависит от используемой конкретной математической модели и технологии изготовления ЦУ. По-видимому, любые статистические данные для оценки упомянутого соотношения вряд ли могут служить основанием для «глобальных» оценок качества алгоритмов, а скорее будут «привязаны» к конкретным ЦУ и носить локальный характер. Поэтому в статье какие-либо данные такого характера не приводятся.

\section{ЗАКЛЮЧЕНИЕ}

В статье предложена процедура нечеткого логического моделирования ЦУ, основанная на применении математического аппарата непрерывной логики для переменных из ограниченного множества вещественных чисел. Показано, что эта процедура по сравнению с процедурой, базирующейся на использовании известной типовой структуры нечеткой модели ЦУ, значительно менее трудоемка.

\section{Библиографичческий список}

1. Zadeh L. A. Fuzzy sets // Inf. Control. 1965. № 8. P. 338-353.

2. Dubois D., Prade H. Fuzzy numbers, on overview // Analysis of fuzzy information : Mathematics and logic. Boca Raton, FL : CRC Press, 1988. P. 3-39.

3. Kandel A. Fuzzy Mathematical Techniques with Applications. Boston, MA, USA : Addison Wesley Longman Publishing Co., Inc., 1986. 274 p.

4. Kaufman A., Gupta M. M. Introduction to fuzzy arithmetic theory and applications. N.Y. : Van Nostrand Reinhold Co., 1991. 351 p.

5. Hanss $M$. Applied fuzzy arithmetic: Introduction with engineering applications. Berlin ; Heidelberg : Springer Publ. Co., Inc., 2010. 274 p.
6. Яблонский С. В. Введение в дискретную математику : учеб. пособие для вузов. 2-е изд. М. : Наука; Гл. ред. физ.-мат. лит., 1986. 384 с.

7. Скобцов Ю. А., Сперанский Д. В., Скобцов В. Ю. Моделирование, тестирование и диагностика цифровых устройств. М. : Национальный Открытый Университет «ИНТУИТ», 2012. 439 с.

8. Закревский А. Д., Поттосин Ю. В., Черемисинова Л. Д. Логические основы проектирования дискретных устройств. М. : Физматлит, 2007. 590 с.

9. Левин В. И. Динамика логических устройств и систем. М. : Энергия, 1980. 224 с.

10. Левин В. И. Бесконечнозначная логика в задачах кибернетики. М. : Радио и связь, 1982. 176 с. 
12. Пегат $A$. Нечеткое моделирование и управление : пер. с англ. 2-е изд. М. : БИНОМ ; Лаборатория знаний, 2013. 798 с.

\section{An Approach to Fuzzy Modeling of Digital Devices}

\section{V. Speranskiy}

Speranskiy Dmitriy Vasilievich, Moscow University of Railway Engineering, 22/2, Chasovaja st., Moscow, Russia, 125993, Speranskiy.dv@gmail.com

In the article the problem of fuzzy binary logic modeling for digital devices (DD) is investigated. In contrast to the similar classic problem of logical simulation, it is assumed that inputs signals of DD are fuzzy signals. In the real of DD for each input ( 0 or 1$)$ there is a certain voltage range. If an input signal is out of the range, the correct signal identification is not guaranteed. The fuzziness of input signals means that there observed values can be either within of the defined range, or out of it. It is known that the logic modeling of every DD is the calculation of value of the certain logical expression. This expression is a mathematical model of DD. Also, the corresponding expression can be always represented in the terms of three logic operations, namely, AND, OR, and NOT. In article, a method of reducing the investigated problem to the problem of fuzzy modeling systems in the space of real numbers is proposed. The method is based on the presentation of logical expression using the infinite-valued (continuous) logic. The calculations in this logic are reduced to the evaluation of the expression in the space of real numbers. The proposed procedure in article is much less labor intensive than the previously known procedure for fuzzy modeling using fuzzy arithmetic in the space of real numbers.

Key words: continuous logic, digital devices, fuzzy logic modeling.

\section{References}

1. Zadeh L. A. Fuzzy sets. Inf. Control, 1965, no. 8, pp. 338-353.

2. Dubois D., Prade H. Fuzzy numbers, on overview. Analysis of fuzzy information: Mathematics and logic. Boca Raton, FL, CRC Press, 1988, pp. 3-39.

3. Kandel A. Fuzzy Mathematical Techniques with Applications. Boston, MA, USA, Addison Wesley Longman Publishing Co., Inc., 1986, 274 p.

4. Kaufman A., Gupta M. M. Introduction to fuzzy arithmetic theory and applications. New York, Van Nostrand Reinhold Co., 1991, 351 p.

5. Hanss M. Applied fuzzy arithmetic: Introduction with engineering applications. Berlin ; Heidelberg, Springer Publishing Co., Inc., 2010, 274 p.

6. Yablonskiy S. V. Vvedenie v diskretnuyu matematiku [Introduction to discrete mathematics]. Moscow, Nauka, 1986, 384 p. (in Russian).

7. Skobtsov Yu. A., Speranskiy D. V., Skobtsov V. Yu. Modelirovanie, testirovanie $i$ diagnostika tsifrovykh ustroystv [Modeling, testing and diagnostics of digital devices]. Moscow, National Public University “INTUIT” Publ., 2012, 439 p. (in Russian).
8. Zakrevskiy A. D., Pottosin Yu. V., Cheremisinova L. D. Logicheskie osnovy proektirovaniya diskretnykh ustroysto [Logic design principles of discrete devices]. Moscow, Fizmatlit, 2007, 590 p. (in Russian).

9. Levin V. I. Dinamika logicheskih ustroistv $i$ sistem [The Dynamics of Logic Units and Systems]. Moscow, Energiya, 1980, 224 p. (in Russian).

10. Levin V. I. Beskonechnoznachnaya logika v zadachah kibernetiki [Infinite-Valued Logic in Problems of Cybernetics]. Moscow, Radio i Svyaz', 1982, 176 p. (in Russian).

11. Ginzburg S. A. Matematicheskaya nepreryonaya logika $i$ izobrazheniye funktsiy [Mathematical continuous logic and the representation of functions]. Moscow, Energiya, 1968, 136 p. (in Russian).

12. Piegat A. Fuzzy Modeling and Control. Springer Science \& Business Media, 2001, 728 p. (Rus. ed. : Piegat A. Nechetkoe modelirovanie i upravlenie. Moscow, BINOM, Laboratoriya znaniy Publ., 2013, 798 p.) 